\title{
Cytokine profile associated with chronic and acute human schistosomiasis mansoni
}

\author{
Clarice Neuenschwander Lins de Morais $/{ }^{+}$, Joelma Rodrigues de Souza, Wlademir Gomes Melo, \\ Marcílio L Aroucha', Paulo Miranda', Ana Lúcia Coutinho Domingues' ${ }^{1}$, \\ Frederico Guilherme Coutinho Abath $^{\dagger}$, Silvia Maria Lucena Montenegro
}

Departamento de Imunologia, Centro de Pesquisas Aggeu Magalhães-Fiocruz, Av. Morais Rego s/nº, Cidade Universitária, 50670-420 Recife, PE, Brasil ' Universidade Federal de Pernambuco, Recife, PE, Brasil

The production and regulation of interleukin (IL) IL-13, IL-4 and interferon-gamma (IFN- $\gamma$ ) was evaluated in 43 schistosomiasis patients with different clinical forms. Whole-blood cultures cytokine production in response to soluble egg antigen (SEA), soluble worm adult preparation (SWAP), mitogens, neutralizing antibodies or recombinant IL-13 were measured by ELISA. After SWAP stimulation, chronic patients, particularly hepatointestinals, produced higher levels of IL-4 in comparison with acute patients, suggesting the presence of a type 2 cytokine profile in these patients. Following SEA and SWAP stimulation, hepatosplenic (HS) patients showed increased levels of IFN- $\gamma$ when compared with acute patients, indicating that HS disease in humans is associated with a type 1 cytokine response. The mechanisms of immune regulation are apparently different between the clinical stages of the disease, some of which are antigen-specific.

Key words: Schistosoma mansoni - cytokines - human schistosomiasis

Granuloma formation and fibrosis are the major causes of morbidity and mortality in association with schistosomiasis. Although granuloma formation is beneficial for the host because it blocks the hepatotoxic effects of antigen released from parasite eggs, this process may lead to fibrosis with excessive accumulation of collagen and other extracellular matrix proteins in the periportal space (Henri et al. 2002). Most infected people living in highly endemic areas are able to control schistosomiasis infection and only have a few pathological manifestations. Unfortunately, a small percentage of infected people develop fibrosis that is associated with granuloma formation, which can lead to portal hypertension. This can result in the formation of esophageal varices, digestive bleeding and sometimes death (Henri et al. 2002). In humans, it is still not clear which cytokines are involved in the pathology of schistosomiasis mansoni.

In the murine model of schistosomiais, type $1 \mathrm{cy}-$ tokines such as interferon- $\gamma$ (IFN- $\gamma$ ) and activated macrophages have been correlated with immunity. On the other hand, type 2-associated cytokines such as interleukin (IL), IL-4, IL-13 and IL-10 inhibit classical macrophage activation and have been implicated in granuloma formation and fibrogenesis around tissue-deposited eggs (Wynn \& Cheever 1995, Hesse et al. 2001, Morais

\footnotetext{
† in memoriam

Financial support: Fiocruz and NIH

+ Corresponding author: clarice@cpqam.fiocruz.br

Received 15 April 2008

Accepted 8 September 2008
}

et al. 2002). Most importantly, it has been observed that IL-13, but not IL-4, is the major type 2 cytokine driving type I and III collagen mRNA production and hepatic fibrosis in infected mice (Wynn et al. 1993, Chiaramonte et al. 1999a). In the context of the type 1/type 2 cytokine paradigm, data from mice and humans have categorized schistosomiasis as a predominantly type 2 disease (Wynn \& Cheever 1995, Abbas et al. 1996, Allen \& Maizels 1997, Fallon 2000, Morais et al. 2002). A type 2 response generally causes morbidity and is detrimental to the host. In fact, infection of humans with schistosomes and other helminths is characteristically associated with elevated $\operatorname{IgE}$ and eosinophilia, which are hallmarks of a type 2 cytokine response (Abbas et al. 1996). This concept has resulted in a body of research that has attempted to prevent putative type 2 responses and ultimately reduce morbidity. However, recent data generated in the murine model suggests that the original distinct categorization of schistosome pathology being caused by type 2 cytokines is misleading. More convincing evidence from schistosome-infected humans suggests that a pro-inflammatory type 1 response is the real cause of morbidity (Fallon 2000, Morais et al. 2002). Previous studies have shown that the pattern of cytokine production changes at different stages of human schistosomiasis. Moreover, it was more specifically demonstrated that early infection is associated with a significant IFN- $\gamma$ response and IL-10 plays an important downregulatory role in that response during late infection (Montenegro et al. 1999). The disease is more severe when the immunopathological mechanisms are dominant or effective control of the parasite is not attained (Chensue et al. 1997). However, the mechanisms responsible for the transition to the most severe forms of the disease are not clear. The understanding of the immunopathol- 
ogy of schistosomiasis has progressed through the use of murine and non-human primate animal models. Nevertheless, studies in humans are relatively rare.

In the present work, the cytokines IL-4, IL-13 and IFN- $\gamma$ along with some regulatory mechanisms were studied with the aim of understanding their possible role in different clinic forms of human schistosomiasis mansoni.

\section{SUBJECTS, MATERIALS AND METHODS}

Subject selection - Chronic schistosomiasis patients were clinically classified as: intestinal (IN) $(\mathrm{n}=11)$, hepatointestinal $(\mathrm{HI})(\mathrm{n}=10)$ and hepatosplenic $(\mathrm{HS})(\mathrm{n}=$ 10) and selected at the ambulatory of Hospital das Clínicas, Universidade Federal de Pernambuco, Brazil. All HS patients were compensated forms of HS disease that had not received hospital care.

Because of a recent outbreak of acute schistosomiasis at Porto de Galinhas, Northeast Brazil, acute patients $(\mathrm{n}=12)$ were selected for this study. These patients had the clinical symptoms of acute disease (fever, arthralgias, diarrhea, hepatosplenomegaly) and upon examination were found to have Schistomosm mansoni eggs in their stool and eosinophilia according to laboratory tests (Barbosa et al. 2001).

Clinical, 03 parasitological stool examinations, total $\mathrm{IgE}$, eosinophils and leukocyte levels were determined for all study groups. All patients had blood samples collected prior to specific treatment with oxamniquine $(15 \mathrm{mg} /$ $\mathrm{kg}$ ) and blood samples from acute patients were collected 60 days after exposure. Patients were aged 6-27 years, $60.5 \%$ of patients were male and $39.5 \%$ of patients were female (Table). Ten out of 31 chronic patients had other intestinal parasitic infections such as Ascaris lumbricoides and Trichuris trichiura and were treated prior to this study.

Ultrasonographic examination was performed on five $\mathrm{HI}$ and eight $\mathrm{HS}$ patients. In two HI patients had aliver with periportal fibrosis degree I and in three had a liver wity periportal fibrosis degree II. All eight HS patients showed periportal fibrosis degree II.

The control group $(\mathrm{n}=10)$ was from non-endemic areas of Pernambuco, Brazil and had three negative parasitological stool examination and no history of contact with contaminated water. All patients and guardians of minors signed an informed consent form and the study was reviewed and approved by the Ethical Committee of the Centro de Pesquisas Aggeu Magalhães da Fundação Oswaldo Cruz, Recife, Pernambuco, Brazil.

Culture conditions - Peripheral blood was drawn with heparin $(10 \mathrm{U} / \mathrm{mL})$ and diluted immediately in RPMI 1640 medium (1:3) plus penicillin (100 U/mL) and streptomycin $(100 \mu \mathrm{g} / \mathrm{mL})$. The whole blood cultures were stimulated as previously described (Montenegro et al. 1999). Parasite antigens, mitogens, antibodies and recombinant cytokines were added to the appropriate wells. Soluble egg antigen (SEA) and soluble adult worm preparation (SWAP) were used at a final concentration of $20 \mu \mathrm{g} / \mathrm{mL}$ and $50 \mu \mathrm{g} / \mathrm{mL}$, respectively (Boros \& Warren 1970, Pearce et al. 1992). Phorbol myristate acetate $(50 \mathrm{ng} / \mathrm{mL})$ plus ionomycin $(1 \mu \mathrm{g} / \mathrm{mL})$ were used as mitogens. The action of the cytokine IFN- $\gamma$ was neutralized by antibodies against this cytokine and its receptor $(\alpha \mathrm{IFN} \gamma / \mathrm{IFN} \gamma \mathrm{R}, 7 \mu \mathrm{g} / \mathrm{mL})$. To analyze the effects of the cytokine IL-13, we added recombinant IL-13 (rIL-13) to the cultures so that the final concentration was $20 \mathrm{ng} / \mathrm{mL}$ and neutralizing antibodies ( $\alpha$ IL-13) were used at a final concentration of $5 \mu \mathrm{g} / \mathrm{mL}$. After $96 \mathrm{~h}$ incubation at $37^{\circ} \mathrm{C}$ in a humidified atmosphere with $5 \% \mathrm{CO}_{2}$ the supernatants were collected and stored at $-70^{\circ} \mathrm{C}$ for subsequent determination of cytokine production.

Cytokine level determination - The levels of IFN- $\gamma$ and IL-4 were measured by enzyme linked immunosorbent assay (ELISA) using specific capture and detection monoclonal antibodies as previously described (Montenegro et al. 1999). IL-13 production was measured using Quantikine Human IL-13 Immunoassay (R \& D systems), following the manufacturer's instructions.

Statistical methods - Characteristics (age, gender and stool examinations) of the study subjects were compared using the Mann-Whitney test. The data regarding cytokines levels were tested for normality using the Kolmogorov-Smirnov test. The results were compared using a logarithmic scale of cytokine concentration, which had a normal distribution, by paired or unpaired Student's $t$

TABLE

Demographic data for schistosomiasis study subjects

\begin{tabular}{|c|c|c|c|c|c|}
\hline & \multicolumn{5}{|c|}{ Clinical forms ${ }^{\mathrm{a}}$} \\
\hline & \multicolumn{3}{|c|}{ Acute } & \multicolumn{2}{|c|}{ Chronic } \\
\hline & Control & & Intestinal & Hepatointestinal & Hepatosplenic \\
\hline Male/Female & $2 / 9$ & $7 / 5$ & $6 / 5$ & $7 / 3$ & $6 / 4$ \\
\hline Total & 11 & 12 & 11 & 10 & 10 \\
\hline \multicolumn{6}{|l|}{ Age (yr) } \\
\hline Mean & 36.3 & 13.6 & 10 & 12.3 & 17.6 \\
\hline Range & $(22-56)$ & $(8-20)$ & $(6-18)$ & $(6-19)$ & $(10-27)$ \\
\hline \multicolumn{6}{|c|}{ Kato-Katz (eggs/g feces) } \\
\hline Mean & 0 & 248 & 148 & 595 & 178 \\
\hline Range & & $(24-1.632)$ & $(24-696)$ & $(24-2.160)$ & $(0-816)$ \\
\hline
\end{tabular}

$a$ : all the demographic data were analyzed by Mann-Whitney test; $\mathrm{p}>0.05$. 
test $(\mathrm{p}<0.05$ was considered statistically significant). These statistical analyses were performed using the software Staview 5 for Windows (SAS Institute, Inc, 1999).

\section{RESULTS}

We did not find any differences among the studied groups with regards to demographic features such age, gender and stool examinations (Table; $\mathrm{p}>0.05$ ).

Production of IFN- $\gamma, I L-13, I L-4$ in response to $S E A$ or SWAP stimulation - Following SEA or SWAP stimulation, there was higher IFN- $\gamma$ production in chronic HS patients in comparison to acute patients, and this observation was statistically significant (Fig. $1 \mathrm{~A} ; \mathrm{p}=0.041$; Fig. 1B; $p=0.012$ ).

Unexpectedly, the control group had high IFN- $\gamma$ levels in response to SEA or SWAP. This indicated that this group could be sensitized by a different mechanism of exposure to schistosomiasis antigen, since they had a negative stool examination, no clinical symptoms and had not been in contact with infected water.
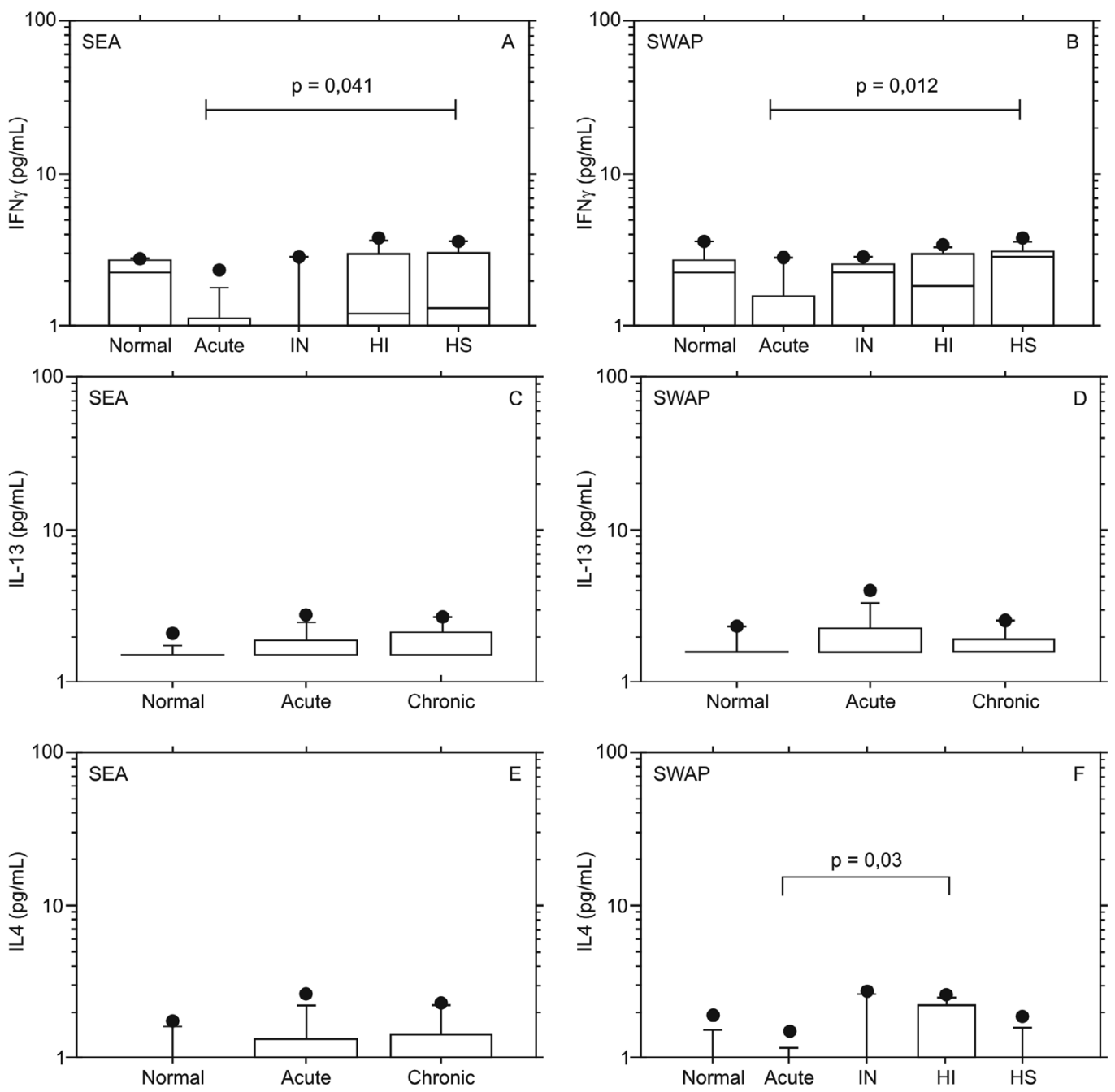

Fig. 1: box plot quartiles and medians of the log of cytokines [A, B: interferon- $\gamma$ (IFN- $\gamma$ ); C, D: interleukin (IL-13); E, F: IL-4] production in response to soluble egg antigen (SEA) or soluble worm adult preparation (SWAP) antigen stimulated whole blood cells supernatants from patients with acute and chronic (HI: hepatointestinal; HS: hepatosplenic; IN: intestinal) schistosomiasis. Significant differences was considered when $\mathrm{p}<0.05$.
The production of IL-13 in schistosomiasis patients stimulated by SEA and SWAP was evaluated and no statistical difference was found in comparison to normal individuals (Fig. 1C, D; $\mathrm{p}>0.05$ ).

Production of IL-4 in schistosomiasis patients after SEA or SWAP antigen stimulation did not differ significantly from normal individuals (Fig. 1E, F; $\mathrm{p}>0.05$ ). However, after SWAP stimulation, HI patients produced higher levels of IL-4 in comparison with acute patients (Fig. 1F; $\mathrm{p}=0.03$ ).

Effects of neutralization of IFN- $\gamma$ and IL-13 on the expression of IL-13, IL-4 and IFN- $\gamma$ - After IFN- $\gamma /$ IFN- $\gamma \mathrm{R}$ neutralization plus stimulation with SWAP, chronic patients, particularly HI, expressed higher levels of IL-13 than control individuals (Fig. 2B; $p=0.004$ ) and patients with the acute form of schistosomiasis (Fig. 2B; $p=0.045)$. The significant increase in IL-13 production in SWAP-stimulated patients after IFN- $\gamma$ neutralization indicated the presence of Th1/Th2 cross-regulation.

when $p<0.05$. 

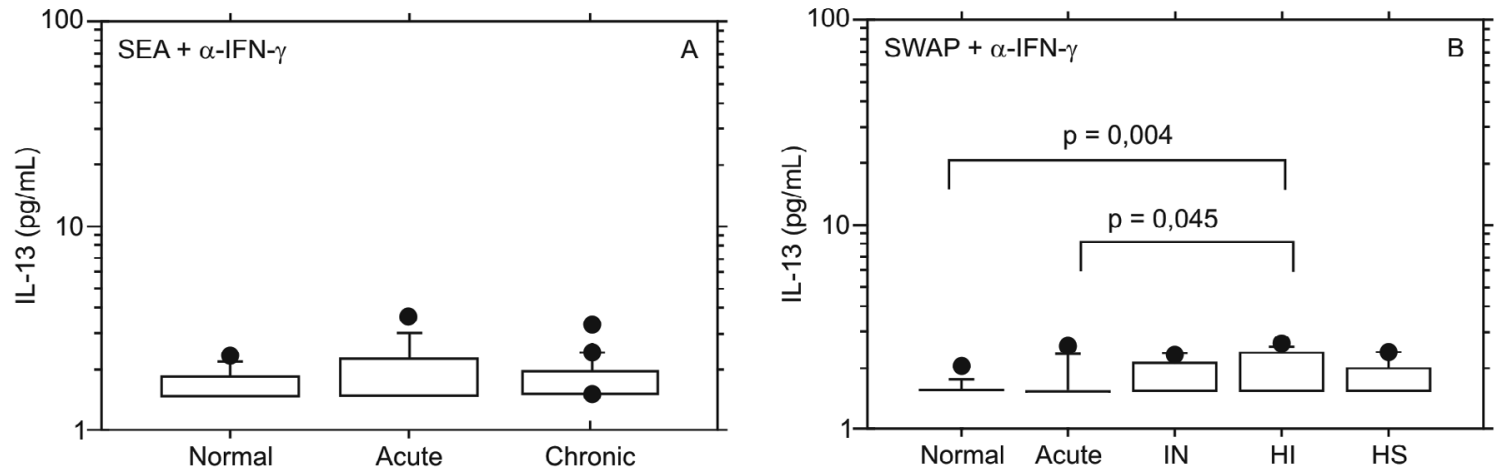

Fig. 2: box plot quartiles and medians of the log of interleukin (IL-13) cytokine production in response to soluble egg antigen (SEA) + neutralizing antibodies against interferon- $\gamma($ IFN- $\gamma)$ and its receptor $(\alpha$ IFN- $\gamma /$ IFN $\gamma \mathrm{R})(\mathrm{A})$ or SWAP (soluble worm adult preparation) $+\alpha$ IFN- $\gamma /$ IFN $\gamma \mathrm{R}$ (B) stimulated whole blood cells supernatants from patients with acute and chronic (HI: hepatointestinal; HS: hepatosplenic; IN: intestinal) schistosomiasis. Significant differences was considered when $\mathrm{p}<0.05$.
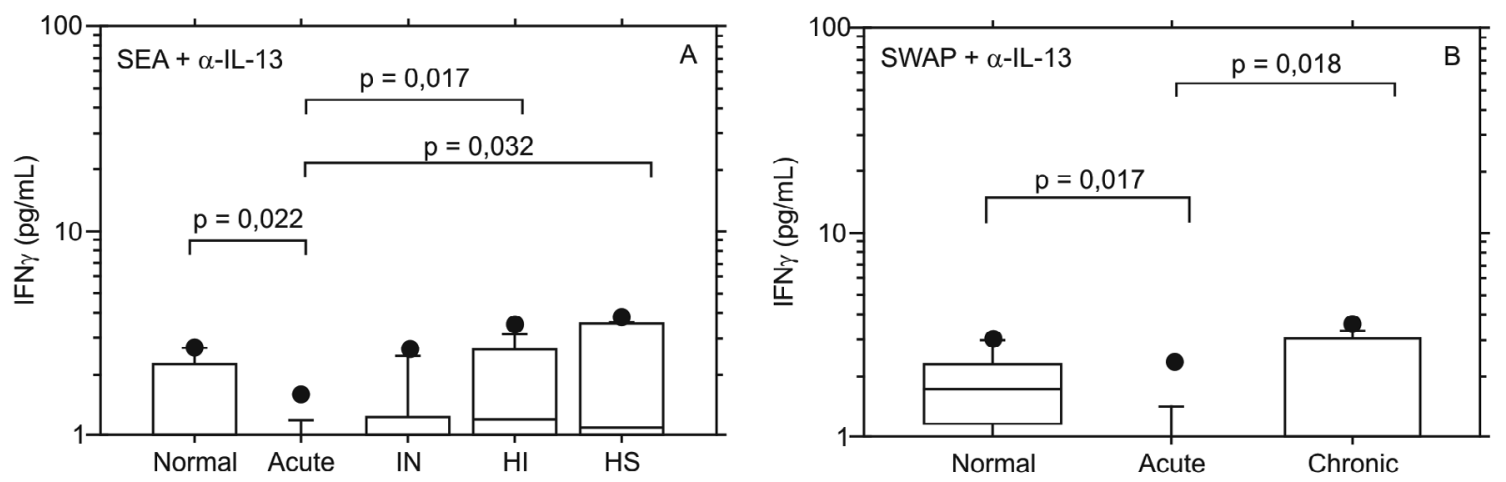

Fig. 3: box plot quartiles and medians of the log of interferon- $\gamma($ IFN- $\gamma$ ) cytokine production in response to soluble egg antigen (SEA) + neutralizing antibodies against interleukin $\alpha$ IL-13 (IL-13) (A) or soluble worm adult preparation (SWAP) + IL-13 (B) stimulated whole blood cells supernatants from patients with acute and chronic (HI: hepatointestinal; HS: hepatosplenic; IN: intestinal) schistosomiasis. Significant differences was considered when $\mathrm{p}<0.05$.

Following IL-13 neutralization and antigen stimulation with SEA $(p=0.022)$ or SWAP $(p=0.017)$, we observed decreased production of IFN- $\gamma$ by acute patients in comparison with the control group. However, higher production of IFN- $\gamma$ was observed in chronic HI $(\mathrm{p}=0.017)$ and HS $(p=0.032)$ patients in comparison with acute patients after IL-13 neutralization plus SEA stimulation. Chronic patients had higher levels of IFN- $\gamma$ in comparison with acute patients after IL-13 neutralization plus SWAP stimulation ( $p=0.018$ ). These observations suggest that there are regulatory elements that control cytokine levels at different clinical stages of the disease and with stimulation by different antigens (Fig. 3A, B).

In addition, $\mathrm{HI}$ patients showed higher production of IL-4 under two conditions: when compared with IN patients after stimulation with SEA plus IFN- $\gamma / \mathrm{IFN}-\gamma \mathrm{R}$ neutralization (Fig. 4A; $p=0.013$ ) and when compared with acute patients after stimulation with SWAP plus IFN- $\gamma /$ IFN- $\gamma$ R neutralization (Fig. 4B; $p=0.04$ ).

IN patients produced significant higher levels of IL-4 in comparison with acute patients in response to IL-13 neutralization plus SWAP stimulation (Fig. $4 \mathrm{D} ; \mathrm{p}=0.015$ ).
Effects of exogenous IL-13 on IL-4 and IFN- $\gamma$ production - Schistosomiasis acute patients, produced an increased amount of IL-4 after SWAP plus rIL-13 stimulation in comparison with antigen stimulation only (Figs $5 \mathrm{~B}, 1 \mathrm{~F} ; \mathrm{p}=0.03$ ).

Addition of rIL-13 to cultures of schistosomiasis patients stimulated with SEA resulted in higher expression of IFN- $\gamma$ in acute patients in comparison to the same patients under SEA stimulation only (Figs 6A, $1 \mathrm{~A} ; \mathrm{p}=0.027$ ). However, after combined stimulation with SWAP and recombinant IL-13, chronic I and HI patients produced lower levels of IFN- $\gamma$ when compared with the same patients stimulated with SWAP only (Figs 6B, 1B; $\mathrm{p}<0.001$ ).

Total IgE and eosinophil levels - Total IgE plasma levels were measured in all 43 schistosomiasis patients. HS $(p=0.004)$ and HI $(p=0.005)$ patients produced higher levels of total IgE than acute patients. In addition, acute patients produced significantly higher IgE levels than the control group (Fig. 7A; $p=0.005$ ).

No significant differences were found in the eosinophilia percentage among chronic and acute schistosomiasis patients (Fig. 7B). 

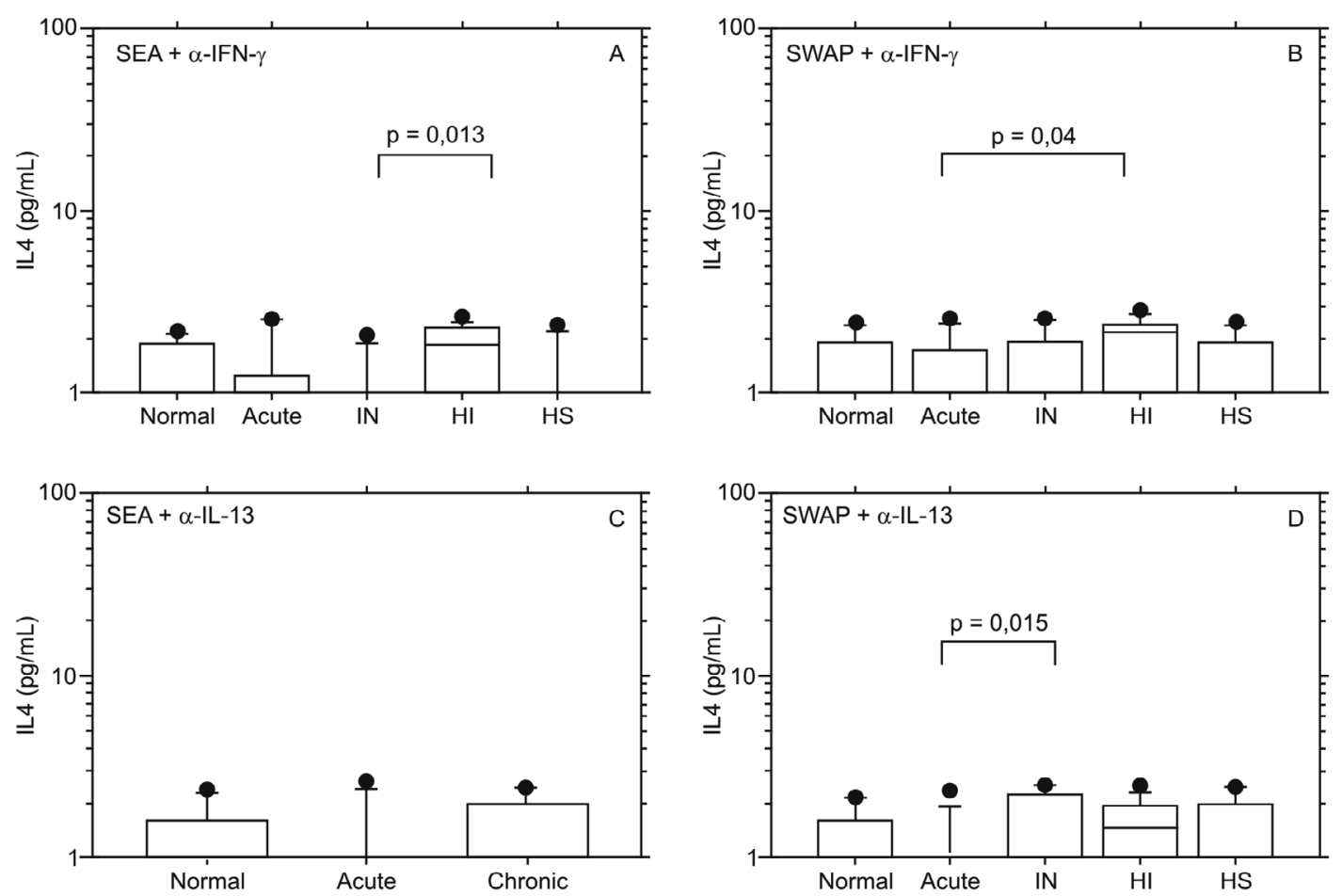

Fig. 4: box plot quartiles and medians of the log of interleukin (IL-4) cytokine production in response to SEA (soluble egg antigen) + neutralizing antibodies against interferon- $\gamma($ IFN $-\gamma$ ) and its receptor $(\alpha \operatorname{IFN}-\gamma /$ IFN $\gamma \mathrm{R})$ (A), SWAP (soluble worm adult preparation) $+\alpha$ IFN- $\gamma /$ IFN $\gamma \mathrm{R}$ (B), SEA + neutralizing antibodies against IL-13 ( $\alpha$ IL-13) (C) or SWAP $+\alpha$ IL-13 (D) stimulated whole blood cells supernatants from patients with acute and chronic (HI: hepatointestinal; HS: hepatosplenic; IN: intestinal) schistosomiasis. Significant differences was considered when $\mathrm{p}<0.05$.
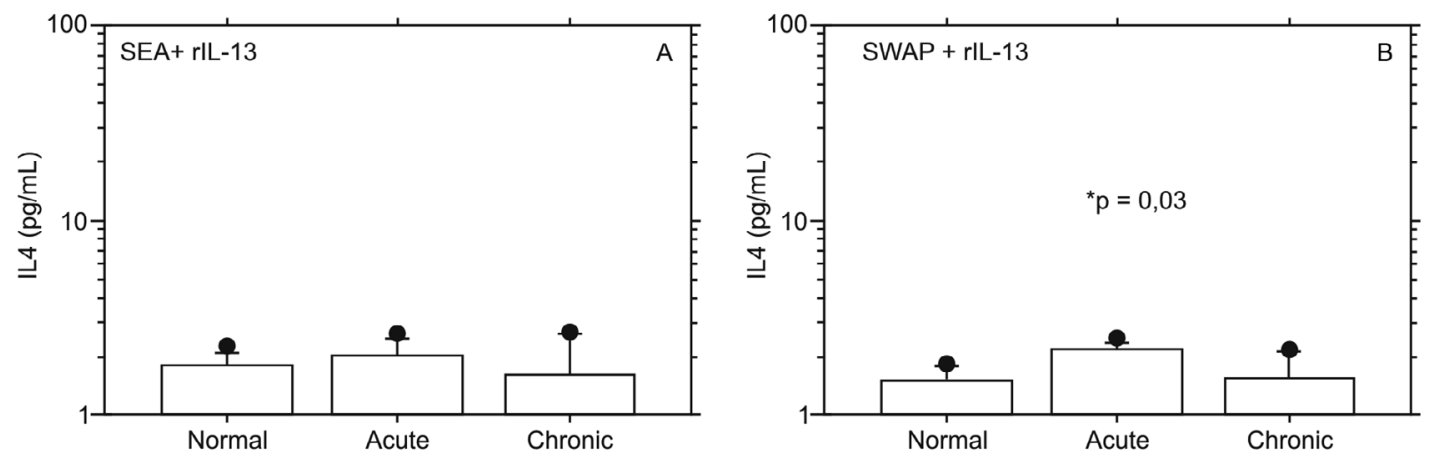

Fig. 5: box plot quartiles and medians of the log of interleukin (IL-4) cytokine production in response to SEA + recombinant IL-13 (rIL-13) (A), SWAP + rIL-13 (B), stimulated whole blood cells supernatants from patients with acute and chronic (HI: hepatointestinal; HS: hepatosplenic; IN: intestinal) schistosomiasis. Significant differences was considered when $\mathrm{p}<0.05$. Asterisk indicates statistic difference between acute patients under SWAP + rIL-13 (B) stimulation in comparison with the same patients under SWAP (Fig.1F) stimulation only.

\section{DISCUSSION}

Both type 1 and type 2 cytokine profiles are involved in granulomatous inflammatory responses. However type 1 (IFN- $\gamma$ ) cytokines and type 2 (IL-4 and IL-13) cytokines exhibit opposing roles in human schistosomiasis mansoni (Morais et al. 2002, Abath et al. 2006).

The role of IL-4, IL-13 and IFN- $\gamma$ in granuloma and fibrosis has been studied in experimental murine models of schistosomiasis (Czaja et al. 1989, Wynn et al. 1993). IFN- $\gamma$ was the major downregulator of fibrosis, whereas IL-4 was shown to play the role of pro-inflammatory cytokine (Chensue et al. 1997), and IL-13 was implicated in fibrogenesis (Chiaramonte et al. 1999a, b, Finkelman et al. 1999). However, it is not clear how much of the data generated with experimental murine models can actually be applied to human disease (Abath et al. 2006).

In humans, the anti-fibrogenic cytokine IFN- $\gamma$ inhibits the production of extracellular matrix proteins by stellate cells and increases collagenase activity in the liver. A genetic study suggested that IFN- $\gamma$ is been associated with protection against severe portal fibrosis. The study mapped a locus on chromosome 6 which has been linked to a high risk of severe hepatic fibrosis, close to the IFN- $\gamma \mathrm{R}$ gene (Dessein et al. 1999, Booth et al. 2004). Moreover, 

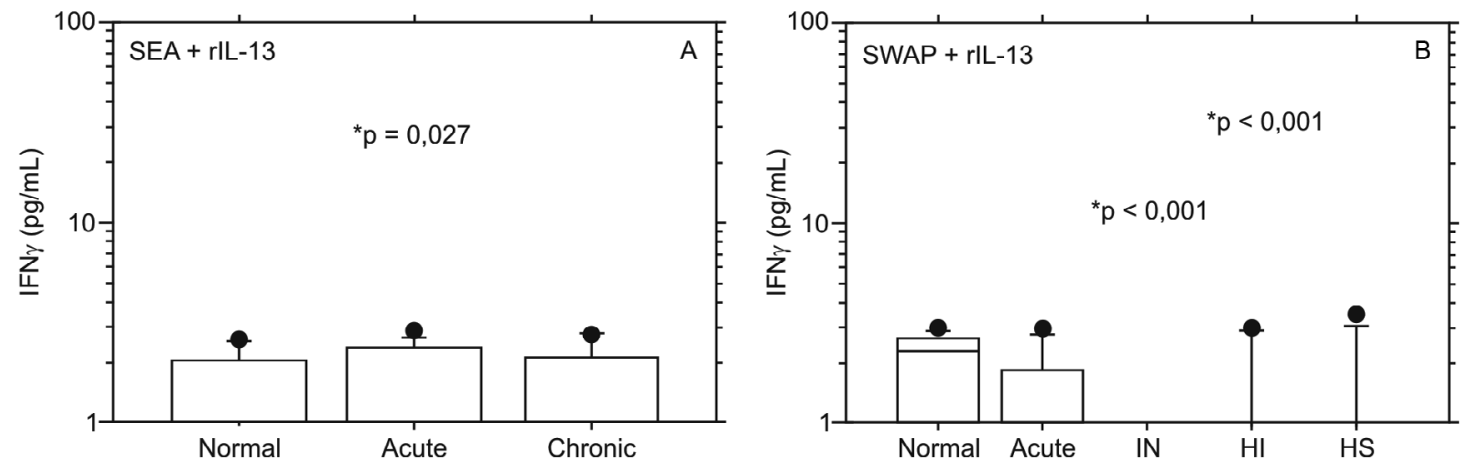

Fig. 6: box plot quartiles and medians of the log of interferon- $\gamma($ IFN- $\gamma$ ) cytokine production in response to SEA + recombinant interleukin (rIL-13) (A), SWAP + rIL-13 (B), stimulated whole blood cells supernatants from patients with acute and chronic (HI: hepatointestinal; HS: hepatosplenic; IN: intestinal) schistosomiasis. Significant differences was considered when $p<0.05$. Asterisks indicate statistic difference between acute patients under SEA + rIL-13 (A) stimulation in comparison with the same patients under SEA stimulation only and between IN and HI patients under SWAP + rIL-13 (B) stimulation in comparison with the same patients under SWAP stimulation only.
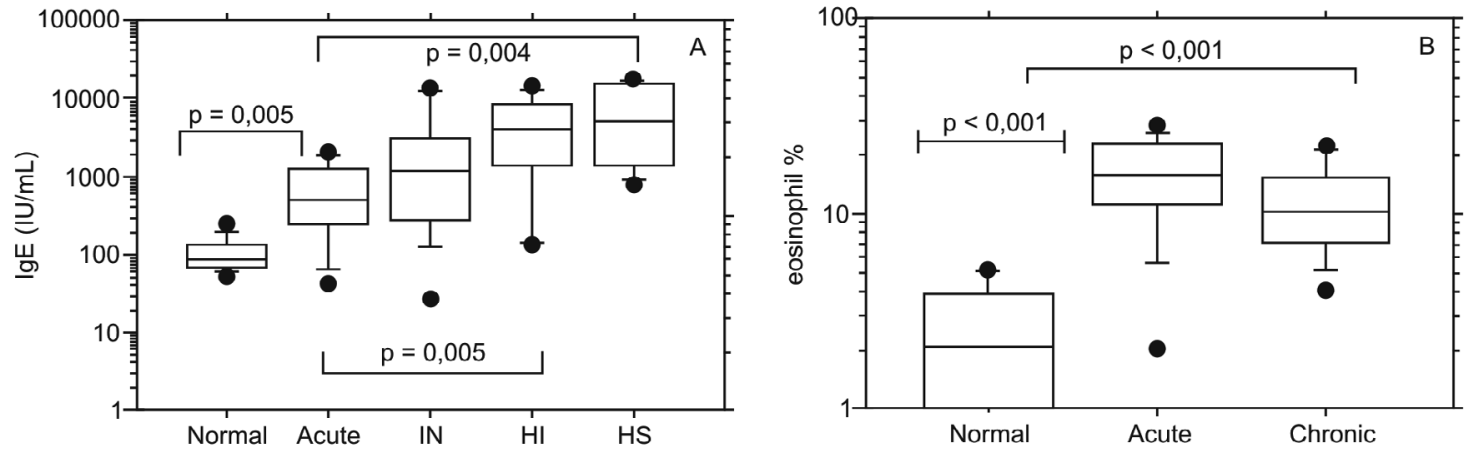

Fig. 7: box plot quartiles and medians of the $\log$ total $\operatorname{IgE}(\mathrm{A})$ and eosinophil levels (B) from patients with acute and chronic (HI: hepatointestinal; HS: hepatosplenic: IN: intestinal) schistosomiasis. Significant differences was considered when $\mathrm{p}<0.05$.

cases of severe portal fibrosis were shown to be associated with low levels of IFN- $\gamma$ and high levels of TNF- $\alpha$ (Henri et al. 2002, Booth et al. 2004, Ribeiro de Jesus et al. 2004). Interestingly, IFN- $\gamma$ produced following SEA or SWAP antigen stimulation gradually increased and correlated with the severity of the chronic clinical form.

Our results showed low levels of IFN- $\gamma$ production in infected patients with the IN clinical form of schistosomiasis (Zwingenberger et al. 1991, Viana et al. 1994, Silveira et al. 2004). When compared to acute patients, we found higher IFN- $\gamma$ levels detected in whole blood cultures stimulated with SEA or SWAP antigens from HS patients, which was also associated with degree II of hepatic fibrosis. This suggests that IFN- $\gamma$ levels increase in order to control the progression of hepatic fibrosis in chronic patients. In a recent study, we demonstrated increased IFN- $\gamma$ production in response to SEA and SWAP stimulation after oxamniquine treatment in acute schistosomiasis patients (Souza et al. 2007). In the present study, we analyzed IFN- $\gamma$ production in acute patients before specific treatment.

However, Ribeiro de Jesus et al. (2002) showed higher IFN- $\gamma$ levels in acute patients with the same conditions of infection time of our work and without treatment. Some possible explanations for these contradictory results may include the infection time and treatment, the presence of others factors such as nutritional status, age, gender, genetic variability and parasitaemia that could influence the immune response of these patients.

Following SWAP stimulation, IL-4 levels were higher in chronic patients, mainly HIs, in comparison to acute patients $(\mathrm{p}<0.05)$, indicating that immune responses to schistosome in chronically infected individuals are typically polarized toward Th2 cytokines. This is characterized by eosinophilia and high levels of IgE (Booth et al. 2004, Abath et al. 2006).

At the same time, experimental studies demonstrated that IL-13 has pro-fibrotic activity and could regulate hepatic fibrosis during murine schistosome infection (Chiaramonte et al. 1999b, Fallon et al. 2000). IL-13 induces the production of mRNA for collagen I and III by fibroblasts in the liver. Recently, an IL-13 receptor $\alpha-2$ has been described and may function as a decoy receptor, regulating and blocking IL-13 activity with high affinity and subsequently hepatic fibrosis (Chiaramonte et al. 2003).

Studies in humans showed a correlation between the production of high levels of IL-13 and the development of more severe hepatic fibrosis (Ribeiro de Jesus et al. 
2004). Our study did not detect an association between high levels of IL-13 and HS patients.

Our study showed no significant statistical differences between IL-13 levels in patients with different clinical forms of schistosomiasis following SEA or SWAP stimulation. However, other authors suggested that IL13 levels tend to be higher during the pre-fibrosis phase and can function as an intermittent inducer of fibrosis (Morais et al. 2002, Ribeiro de Jesus et al. 2004).

Interestingly, HI patients had high levels of IL-4 and IL-13 after IFN- $\gamma /$ IFN $\gamma$ R neutralization and SWAP stimulation. These Th2 cytokines (IL-4 and IL-13) are increased during the pre-fibrosis phase, prior to the HS stage. Another important point is that the higher IL-13 levels in HI patients were only observed in IFN- $\gamma / \mathrm{IFN} \gamma \mathrm{R}$ neutralization plus SWAP stimulated cultures in comparison to acute patients (Fig. 2B; $p=0.045$ ) and normal control (Fig. 2B; $p=0.004$ ). In addition, IL-4 levels were increased in HI patients and in SEA-stimulated cultures, suggesting that type 2 responses were observed predominantly after the acute phase and prior to the HS phase of the disease.

Despite the presence of a predominantly type 2 response in infected humans, there is currently no evidence to suggest that immunopathology in humans is driven by type 2 cytokines (Morais et al. 2002). In fact, the available evidence suggests that HS disease in humans is associated with a type 1 cytokine response (Fallon 2000). Mwatha et al. (1998) associated high levels of TNF- $\alpha$ and IFN- $\gamma$ and low levels of IL-5 with the presence of hepatosplenomegaly. Similarly, Montenegro et al. (1999) demonstrated that there was marked production of IFN- $\gamma$, but not of IL-4 and IL-5 by parasite antigen-stimulated splenocytes from $S$. mansoni patients with HS disease. This study showed the contribution of IL-10 in the suppression of a significant IFN- $\gamma$ response in the early and chronic phases of the disease. Falcão et al. (1998) showed that HS schistosomiasis patients produced high levels of IFN- $\gamma$ and low levels of IL-10, associating this cytokine with the control of the development of the severe clinical form. Our study confirmed previously published data that showed that HS schistosomiasis patients are associated with production of high levels of IFN- $\gamma$ have been found in HS schistosomiasis patients.

We found that IL-13 may be inhibiting the production of IFN- $\gamma$ by $\mathrm{T}$ cells of chronic (IN, HI and HS) patients in response to SWAP stimulation. However, in acute patients stimulated with SEA plus exogenous IL-13, there appears to be no inhibitory effect on IFN- $\gamma$ production as evidenced by the increase in IFN- $\gamma$ levels in these patients. Our study strongly suggests that a regulatory mechanism exists between IFN- $\gamma$ and IL-13. This regulation is antigen-specific and different for the various clinical forms of the disease and the mechanisms that control this regulation are still not clear.

The regulation of immunopathology schistosomiasis may be more complex in humans than in experimental models, with multiple mediators capable of regulating the progression of disease (Wilson et al. 2007).
The present study evaluated the cytokine profile in schistosomiasis patients and showed an association between type 1 cytokine IFN- $\gamma$ and the HS clinical form of disease. IFN- $\gamma$ may not be the cause HS disease; rather it may control the progression of severity during this phase of schistosomiasis.

In conclusion, the production and regulation of Th1 and Th2 cytokines appears to change during different clinical stages of human schistosomiasis. This indicates that there is a very complex orchestration of the immune response during the transition to more severe forms of the disease. Future studies must continue to improve approaches to study the human immune response to disease. When this goal is achieved, effective methods of manipulating the immune system, such as vaccination and treatment can be developed.

\section{ACKNOWLEDGEMENTS}

To Dr. Thomas Wynn (National Institute of Allergy and Infectious Diseases, NIH) for your assistance in the beggining of this project.

\section{REFERENCES}

Abath FGC, Morais CNL, Montenegro CEL, Wynn TA, Montenegro SML 2006. Immunopathogenic mechanisms in schistosomiasis: what can be learnt from human studies? Trends Parasitol 22: 85-91.

Abbas AA, Murphy KM, Sher A 1996. Funccional diversity of helper T lymphocytes. Nature 383: 787-793.

Allen JE, Maizels RM 1997. Th1-Th2: reliable paradigm or dangerous dogma? Immunol Today 18: 387-392.

Barbosa CS, Domingues ALC, Abath FGC, Montenegro SML, Guida U, Carneiro J, Tabosa B, Morais CNL, Spinelli V 2001. Epidemia de esquistossomose aguda na praia de Porto de Galinhas, Pernambuco, Brasil. Cad Saúde Públ 17: 725-728.

Booth M, Mwatha JK, Joseph S, Jones FM, Kadzo H, Ireri E, Kazibwe F, Kemijumbi J, Kariuki C, Kimani G, Ouma JH, Kabatereine NB, Vennervald BJ, Dunne DW 2004. Periportal fibrosis in human Schistosoma mansoni infection is associated with low IL-10, low IFN-g, high TNF-alpha, or low RANTES, depending on age and gender. J Immunol 172: 1295-1303.

Boros DV, Warren KS 1970. Delayed hypersensitivity type granuloma formation and dermal reaction induced and elicited by a soluble factor isolated from Schistosoma mansoni eggs. J Exp Med 132: 488-507.

Chensue SW, Warmington K, Ruth JH, Lukacs N, Kunkel S 1997. Mycobacterial and schistosomal antigen-elicited granuloma formation in IFN- $\gamma$ and IL-4 knockout mice. J Immunol 159: 3565-3573.

Chiaramonte MG, Donaldson DD, Cheever AW, Wynn TA 1999a. An IL-13 inhibitor blocks the development of hepatic fibrosis during a $\mathrm{T}$ helper type 2 dominated inflammatory response. J Clin Invest 104: 777-785.

Chiaramonte MG, Mentink-Kane M, Jacobson BA, Cheever AW, Whitters MJ, Goad MEP, Wong A, Collins M, Donaldson MM, Grusby MJ, Wynn TA 2003. Regulation and function of interleukin 13 receptor alpha 2 during T helper cell type 2-dominant immune response. J Exp Med 197: 687-701.

Chiaramonte MG, Schopf LR, Neben TY, Cheever AW, Donaldson DD, Wynn TA 1999b. IL-13 is a key regulatory cytokine for Th2 cell-mediated pulmonary granuloma formation and $\mathrm{IgE}$ responses induced by Schistosoma mansoni eggs. J Immunol 162: 920-930. 
Czaja MJ, Weiner FR, Takahashi S, Giambrone MA, Van der Meide PH, Schellekens H, Biempica L, Zern MA 1989. Gamma Interferon treatment inhibts collagen deposition in murine schistosomiasis. Hepatology 10: 795-800.

Dessein AJ, Hillaire D, Elwali NE, Marquet S, Mohamed-Ali Q, Mirghani A, Henri S, Abdelhameed AA, Saeed OK, Magzoub MM, Abel L 1999. Severe hepatic fibrosis in Schistosoma mansoni infection is controlled by a major locus that is closely linked to the IFN-g receptor gene. Am J Hum Genet 65: 709-721.

Falcão PL, Malaquias LCC, Martins-Filho OA, Silveira AMS, Passos VMA, Prata A, Gazzinelli G, Coffman RL, Correa-Oliveira R 1998. Human schistosomiasis mansoni: IL-10 modulates the in vitro granuloma formation. Parasite Immunol 20: 441-454.

Fallon PG 2000. Immunopathology of schistosomiasis: a cautionary tale of mice and men. Immunol Today 21: 29-35.

Fallon PG, Richardson EJ, McKenzie GJ, McKenzie ANJ 2000. Schistosome infection of transgenic mice defines distinct and contrasting pathogenic roles for IL-4 and IL-13: IL-13 is a profibrotic agent. J Immunol 164: 2585-2591.

Finkelman FD, Wynn TA, Donaldson DD, Urban-Jr JF 1999. The role of IL-13 in helminth induced inflammation and protective immunity against nematode infections. Curr Opin Immunol 11: 420-426.

Henri S, Chevillard C, Mergani A, Paris P, Gaudart J, Camilla C, Dessein H, Montero F, Elwali NMA, Saeed OK, Magzoub M, Dessein AJ 2002. Cytokine regulation of periportal fibrosis in human infected with Schistosoma mansoni: IFN-g is associated with protection against fibrosis and TNF-alpha with aggravation of disease. J Immunol 169: 929.

Hesse M, Modolell M, La Flamme AC, Schito M, Fuentes JM, Cheever AW, Pearce EJ, Wynn TA 2001. Differential regulation of nitric oxide synthase- 2 and arginase- 1 by type 1/type 2 cytokines in vivo: granulomatous pathology is shaped by the pattern of Larginine metabolism. J Immunol 167: 6533-6544.

Montenegro SML, Miranda P, Mahanty S, Abath FGC, Teixeira KM, Coutinho EM, Brinkman J, Gonçalves I, Domingues LAW, Domingues ALC, Sher A, Wynn TA, 1999. Cytokine production in acute versus chronic human schistosomiasis mansoni: the cross-regulatory role of interferon $-\gamma$ and interleukin-10 in the responses of peripheral blood mononuclear cells and splenocytes to parasite antigens. J Infect Dis 179: 1502-1514.

Morais CNL, Souza JR, Melo WG, Aroucha ML, Domingues ALC, Wynn T, Abath FGC, Montenegro SML 2002. Studies on the production and regulation of interleukin, IL-13, IL-4 and Interferon- $\gamma$ in human schistosomiasis mansoni. Mem Inst Oswaldo Cruz 97: 113-114.

Mwatha JK, Kimani G, Kamau T, Mbugua G, Ouma JH, Mumo J, Fulford AJC, Jones FM, Butterworth AE, Roberts MB, Dunne
DW 1998. High levels of TNF- $\alpha$, soluble TNF receptors, soluble ICAM-1, and IFN $-\gamma$, but low levels of IL-5 are associated with hepetosplenic disease in human schistosomiasis mansoni. $J$ Immunol 160: 1992-1999.

Pearce EJ, Caspar P, Gryzch JM, Lewis FA, Sher A 1992. Downregulation of Th1 cytokine production accompanies induction of Th2 responses by a helminth, Schistosoma mansoni. J Exp Med 173: 159-166.

Ribeiro de Jesus A, Magalhães A, Miranda DG, Miranda RG, Araújo MI, de Jesus AA, Silva A, Santana LB, Carvalho EM 2004. Association of type 2 cytokines with hepatic fibrosis in human Schistosoma mansoni infection. Infect Immun 72: 3391-3397.

Ribeiro de Jesus A, Silva A, Santana LB, Magalhães A, de Jesus AA, Almeida RP, Rego MAV, Burattini MN, Pearce EJ, Carvalho EM 2002. Clinical and immunologic evaluation of 31 patients with acute schistosomiasis mansoni. J Infect Dis 185: 98-105.

Silveira AMS, Gazzinelli G, Alves-Oliveira LF, Bethony J, Gazzinelli A, Carvalho-Queiroz C, Alvarez MCB, Lima-Silva FC, Prata A, LoVerde PT, Correa-Oliveira R 2004. Human schistosomiasis mansoni: intensity of infection differentially affects the production of Interleukin-10, interferon- $\gamma$ and interleukin-13 by soluble egg antigen or adult worm antigen stimulated cultures. Trans $R$ Soc Trop Med Hyg 98: 514-519.

Souza JR, Morais CNL, Aroucha ML, Miranda PJC, Barbosa CS, Domingues ALC, Carvalho Júnior LB, Abath FGC, Montenegro SML 2007 Treatment of human acute schistosomiasis with oxamniquine induces an increase in interferon- $\gamma$ response to Schistosoma mansoni antigens. Mem Inst Oswaldo Cruz 102: 225-228.

Viana IRC, Sher A, Carvalho OS, Massara CL, Elói-Santos SM, Pearce EJ, Colley DG, Gazzinelli G, Correa-Oliveira R 1994. Interferon-g production by peripheral blood mononuclear cells from residents of an area endemic for Schistosoma mansoni. Trans R Soc Trop Med Hyg 88: 466-470.

Wilson MS, Mentink-Kane MM, Pesce JT, Ramalingam TR, Thompson R, Wynn TA 2007. Immunopathology of schistosomiasis. Immunol Cell Biol 85: 148-154.

Wynn TA, Cheever AW 1995. Cytokine regulation of granuloma formation in schistosomiasis. Curr Opin Immunol 7: 505-511.

Wynn TA, Eltoum I, Cheever AW, Lewis FA, Gause WC, Sher A 1993. Analysis of cytokine mRNA expression during primary granuloma formation during induced by eggs of Schistosoma mansoni. J Immunol 151: 1430-1440.

Zwingeberger K, Hohmann A, Cardoso de Brito M, Ritter M 1991. Impaired balance of interleukin-4 and interferon-g production in infection with Schistosoma mansoni and intestinal nematodes. Scand J Immunol 34: 234-251. 